\title{
OPEN Transformation of nonencapsulated Streptococcus pneumoniae during systemic infection
}

\author{
Jessica L. Bradshaw ${ }^{1,2}$, Iftekhar M. Rafiqullah'ㄹ, D. Ashley Robinson ${ }^{1}$ \& Larry S. McDaniel ${ }^{1 \bowtie}$
}

Streptococcus pneumoniae (pneumococcus) is a principal cause of bacterial middle ear infections, pneumonia, and meningitis. Capsule-targeted pneumococcal vaccines have likely contributed to increased carriage of nonencapsulated S. pneumoniae (NESp). Some NESp lineages are associated with highly efficient DNA uptake and transformation frequencies. However, NESp strains lack capsule that may increase disease severity. We tested the hypothesis that NESp could acquire capsule during systemic infection and transform into more virulent pneumococci. We reveal that NESp strains MNZ67 and MNZ41 are highly transformable and resistant to multiple antibiotics. Natural transformation of NESp when co-administered with heat-killed encapsulated strain WU2 in a murine model of systemic infection resulted in encapsulation of NESp and increased virulence during bacteremia. Functional capsule production increased the pathogenic potential of MNZ67 by significantly decreasing complement deposition on the bacterial surface. However, capsule acquisition did not further decrease complement deposition on the relatively highly pathogenic strain MNZ41. Whole genome sequencing of select transformants demonstrated that recombination of up to 56.7 kbp length occurred at the capsule locus, along with additional recombination occurring at distal sites harboring virulence-associated genes. These findings indicate NESp can compensate for lack of capsule production and rapidly evolve into more virulent strains.

Streptococcus pneumoniae (pneumococcus) resides harmlessly in the nasopharynx of asymptomatic carriers, but is also a prominent human pathogen that consistently causes mucosal diseases of the upper respiratory system ${ }^{1}$. Noninvasive diseases associated with pneumococcal infections include conjunctivitis, otitis media (OM), and nonbacteremic pneumonia ${ }^{2,3}$. Invasive pneumococcal disease (IPD) can occur when pneumococci disseminate into normally sterile sites such as the blood and meninges to establish bacteremia and bacterial meningitis, respectively ${ }^{2}$. Prevention measures against pneumococcal disease have been implemented for decades and include two commercially licensed vaccines: PNEUMOVAX 23 and Prevnar $13^{4-6}$. Still, the pneumococcus remains a leading cause of bacterial OM, pneumonia, and meningitis even with vaccine implementation ${ }^{7}$. The resilient pathogenicity of pneumococcus in spite of prevention measures is largely due to limitations in the vaccine strategy and genomic plasticity of the pneumococcus. Currently, there are nearly 100 unique pneumococcal serotypes identified, and the current vaccines together target roughly $25 \%$ of the characterized serotypes ${ }^{8}$. Furthermore, selective pressure on vaccine-inclusive serotypes has likely driven capsule switching and positive selection of mutants expressing antigenically distinct capsule structures and of nonencapsulated S. pneumoniae (NESp) that allow escape from antibody-mediated clearance.

NESp cannot be characterized by capsular serotyping since this subpopulation does not express the polysaccharide capsule. Rather, NESp are characterized by the genes present in the capsular polysaccharide biosynthetic $(c p s)$ locus 9 . Strains that encode nonfunctional variants of capsule genes found in encapsulated strains are considered Group I NESp. Strains characterized as Group II NESp lack the genes encoded in the cps locus of encapsulated strains and instead encode virulence-associated genes $p s p K$, aliC, and aliD in the cps locus. Group II NESp can be further divided into so-called null capsule clades (NCC) based on the genes present in the cps locus ${ }^{9}$. NESp strains that encode $p s p K$ are designated NCC1 while strains that encode aliC and aliD are considered NCC2.

The variability of the pneumococcal genome is greatly impacted by horizontal gene transfer (HGT). Pneumococci are naturally competent for transformation and are thus able to import exogenous DNA that is subsequently integrated into the chromosome via homologous recombination ${ }^{10}$. Integrative conjugative elements

${ }^{1}$ Department of Microbiology and Immunology, University of Mississippi Medical Center, Jackson, MS, USA. ${ }^{2}$ Present address: Department of Physiology and Anatomy, University of North Texas Health Science Center, Fort Worth, TX, USA. ${ }^{\square}$ email: Imcdaniel@umc.edu 


\begin{tabular}{|c|c|c|c|c|c|c|}
\hline Strain & Sequence type & Serotype & Transformation contribution & Antibiotic susceptibility & Capsule locus genes & References \\
\hline WU2 & 378 & 3 & Donor (in vivo) & $\mathrm{Erm}^{\mathrm{S}} \mathrm{Tmp}^{\mathrm{S}}$ & $\operatorname{cps} A^{+}$ & 46 \\
\hline LEK06 & 43 & $19 \mathrm{~F}$ & Donor (in vitro) & Str ${ }^{\mathrm{R}}$ & $c p s A^{+}$ & This study \\
\hline MNZ67 & 1464 & NESp & Recipient & $\mathrm{Erm}^{\mathrm{R}}$ & $\begin{array}{l}c p s A^{-} \\
p s p K^{+}\end{array}$ & 9 \\
\hline 67S1 & 1464 & 3 & MNZ67 Transformant & $\mathrm{Erm}^{\mathrm{R}}$ & $\begin{array}{l}p s A^{+} \\
p s p K^{-}\end{array}$ & This study \\
\hline MNZ41 & 6153 & NESp & Recipient & $\mathrm{Tmp}^{\mathrm{R}}$ & $\begin{array}{l}\text { cpsA- } \\
\text { aliC } \\
\text { aliD }^{+}\end{array}$ & 9 \\
\hline $41 \mathrm{~S} 3$ & 6153 & 3 & MNZ41 Transformant & $\operatorname{Tm}^{\mathrm{R}}$ & $\begin{array}{l}\text { cpsA } A^{+} \\
\text {aliC } \\
\text { aliD }^{-}\end{array}$ & This study \\
\hline
\end{tabular}

Table 1. Description of strains used in this study. Capsule genes were determined by polymerase chain reaction (PCR) analysis and verified by whole genome sequencing. NESp, nonencapsulated Streptococcus pneumoniae; Str, streptomycin; Erm, erythromycin; TMP, trimethoprim; S, sensitive; R, resistant.

(ICEs) and phages may also be present in pneumococcal strains and provide additional mechanisms for HGT. Bacterial factors enhancing colonization and virulence, as well as antibacterial resistance and immune evasion, have been acquired through $\mathrm{HGT}^{11,12}$. Furthermore, NESp are associated with multidrug resistance, which may be a consequence of higher recombination frequencies observed in some NESp when compared to encapsulated pneumococci ${ }^{13}$. Interactions between pneumococcal strains during cocolonization of the nasopharynx provide opportunities for intraspecies HGT to occur. NESp have been shown to colonize the nasopharynx as efficiently as encapsulated pneumococci, and genetic exchange between these populations does occur ${ }^{14,15}$.

S. pneumoniae strains are diverse and express various virulence factors that potentiate mucosal and invasive infections $s^{1,16}$. The polysaccharide capsule is a major virulence factor of the pneumococcus ${ }^{1}$. However, the specific impact of virulence factors is dependent upon the host niche being occupied ${ }^{17}$. For instance, capsule has been shown to have varying impacts on disease states. During colonization of the nasopharynx or lungs and in cases of conjunctivitis, capsule expression is reduced or negatively impacts pneumococcal virulence ${ }^{18,19}$. Yet, capsule has also been shown to be an extremely significant virulence factor due to its protective function during invasive disease $^{20}$. NESp colonize the nasopharynx well but are not efficient in establishing invasive disease ${ }^{3,21}$. Thus, capsule acquisition could expand the pathogenic capabilities of NESp.

In a classic experiment, Frederick Griffith first demonstrated that attenuated, rough colonies could be "transformed" into virulent, smooth colonies during murine infections ${ }^{22}$. However, Griffith used a passaged, avirulent strain rather than a naturally nonencapsulated strain as a transformation recipient. Emerging NESp strains encode novel genes and genetic elements in comparison to those of encapsulated strains ${ }^{1}$. Thus, virulenceassociated consequences of genetic transfer and capsule acquisition in some NESp may be far greater than a null capsule mutant regaining capsule. Moreover, NESp that have acquired multiple antibiotic resistance genes may serve as antibiotic resistance reservoirs that greatly threaten treatment options and survival outcomes ${ }^{3}$. In this study, we hypothesized that naturally nonencapsulated strains will be efficiently transformed when under the selective pressure of invasive disease into more virulent strains. The aim of this study was to determine genetic and phenotypic alterations that occur in NESp during in vivo transformation.

\section{Results}

NESp strains are antibiotic resistant and highly transformable. We first determined in vitro antibiotic susceptibilities of donor and recipient pneumococcal strains used for in vivo transformations. The donor strain, encapsulated serotype 3 S. pneumoniae WU2, was sensitive to both erythromycin (Erm) and trimethoprim (Tmp), while recipient NESp strains MNZ41 and MNZ67 were resistant to Tmp or Erm, respectively (Table 1). These two NESp strains are of different multilocus sequence types and have different genes at the cps locus; thus, these strains represent different NESp lineages. Next, we evaluated in vitro transformation efficiencies of NESp recipient strains using genomic DNA isolated from S. pneumoniae LEK06 harboring chromosomally acquired streptomycin resistance. To become competent for transformation, MNZ67 required only competence stimulating peptide (Csp)-1 while MNZ41 required both Csp-1 and Csp-2. MNZ67 had a higher transformation efficiency (204 \pm 46 transformants/ $\mu \mathrm{g}$ DNA) compared to MNZ41 (164 \pm 44 transformants/ $\mu \mathrm{g}$ DNA), but this difference was not statistically significant $(p=0.593$, student T-test).

NESp strains acquire and express capsule during systemic infection. Since MNZ67 and MNZ41 were highly transformable in vitro, we investigated transformation of these strains during a murine model of systemic infection. Heat-killed S. pneumoniae strain WU2 was chosen as a DA donor because of its mucoid capsule phenotype on blood agar plates that would permit the identification of transformants in a mixed culture of nonencapsulated and encapsulated pneumococci. In mice administered an NESp strain and heat-killed WU2 $(\Delta \mathrm{WU} 2)$, no capsule transformants were identified in blood mixtures isolated at $4 \mathrm{~h}$ postinfection (hpi), as all isolated pneumococci displayed a rough phenotype characteristic of nonencapsulated strains. At 24 hpi, smooth transformants were isolated from mice administered either NESp parent strain with $\Delta \mathrm{WU} 2$, indicating that in vivo pneumococcal transformation occurs within the first $24 \mathrm{~h}$. At $24 \mathrm{hpi}$, homogenous mixtures of nonen- 


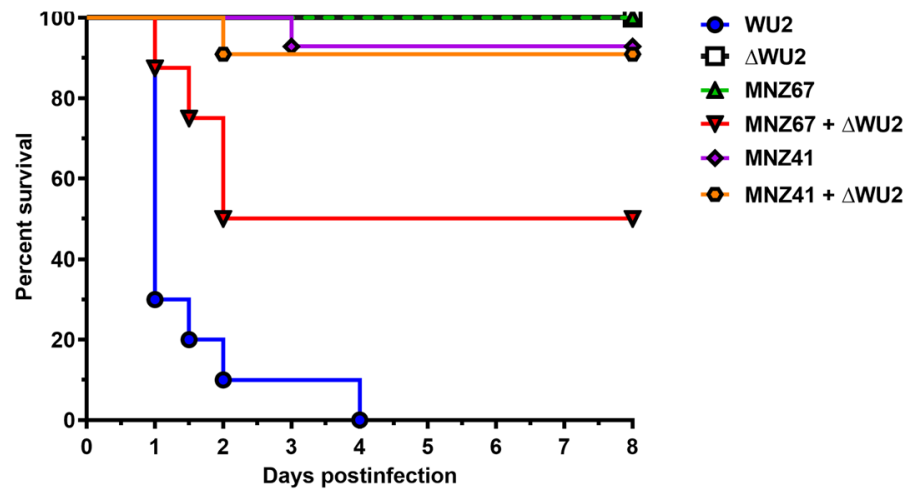

Figure 1. Survival analysis of C57BL/6 mice during pneumococcal bacteremia. Seven-week-old mice were intraperitoneally infected with indicated pneumococcal strains, and survival was monitored over an 8-day period. Survival rates are shown as percentages (WU2: $\mathrm{n}=10, \Delta \mathrm{WU} 2: \mathrm{n}=6$, MNZ67: $\mathrm{n}=8$, MNZ67 $+\Delta W U 2$ : $\mathrm{n}=8$, MNZ41: $\mathrm{n}=14$, MNZ41 $+\Delta \mathrm{WU} 2: \mathrm{n}=11$ ). Significantly higher mortality rates were observed in mice infected with WU2 or MNZ67 $+\Delta \mathrm{WU} 2$ when compared to all other groups. Data are representative of at least two independent experiments. Survival curves were determined to be significantly different $(\mathrm{p}<0.0001)$ by Logrank (Mantel-Cox) test. $\Delta \mathrm{WU} 2=$ heat-killed WU2.

capsulated or encapsulated pneumococci, as well as heterogenous mixtures containing both rough and smooth phenotypes, were isolated from the blood of individual mice administered NESp strains and $\triangle \mathrm{WU} 2$ (Fig. S1). Two in vivo capsule transformants, $41 \mathrm{~S} 3$ and $67 \mathrm{~S} 1$, were selected for screening based on their smooth phenotype on blood agar plates. The genetic basis of this phenotype was initially examined by PCR analysis of the capsular locus. All isolates that appeared to express capsule on blood agar encoded the conserved $\operatorname{cps} A$ gene that replaced $p s p k$ or aliC and aliD encoded in the respective parent strains (Table 1). Furthermore, in vivo transformants that encoded the conserved capsule gene $c p s A$ also retained antibiotic resistance associated with parent (recipient) strains (Table 1).

Capsule acquisition enhances persistence and virulence of transformed NESp during bacteremia. Large variances in bacterial burden occurred in mice that were infected with NESp strain MNZ67 and co-administered $\Delta \mathrm{WU} 2$. In half of the mice administered MNZ67 and $\Delta \mathrm{WU} 2, \mathrm{MNZ67}$ successfully acquired capsule that was verified by culture methods and PCR analysis. In this subset of mice, bacterial burdens were high and resulted in $100 \%$ mortality by 48 hpi (Fig. 1). In the remaining half of the mice administered MNZ67 and $\triangle \mathrm{WU} 2$, there was no evidence of in vivo capsule acquisition. In this subgroup of mice, MNZ67 was rapidly cleared and all mice survived, resulting in an overall mortality of $50 \%$ by 48 hpi (Figs. 1 and 2 ). In mice infected with NESp strain MNZ41, bacterial loads were significantly higher $(p<0.01)$ compared to MNZ67-infected mice from as early as 4 hpi through 72 hpi (Fig. 2). Importantly, MNZ41 capsule acquisition did not significantly enhance murine mortality or bacterial burden when compared to the parent strain (Figs. 1 and 2). Furthermore, NESp MNZ41 was not rapidly cleared during systemic infection (Fig. 2b). However, NESp MNZ41 were cleared from infected mice by 120 hpi while capsule-transformed MNZ41 continued to be isolated from MNZ41-infected mice at 120 hpi (Fig. 2b).

NESp strains acquire virulence factors from an encapsulated strain during bacteremia. The recombination events involving the capsular locus, and the potential for other recombination events outside the capsular locus, were examined by whole genome sequencing. The sequence types of smooth transformants $67 \mathrm{~S} 1$ and 41S3 matched the nonencapsulated parent strains (Table 1), thus confirming the background of the transformants. A single recombination event was identified in the genome of transformant 41S3. The breakpoints of this event occurred within a gene adjacent to $\operatorname{dex} B$ and within the aliA gene, and resulted in the replacement of a 9.9 $\mathrm{kbp}$ fragment with a $13.5 \mathrm{kbp}$ fragment that included the serotype 3 capsular genes (Fig. $3 \mathrm{a}$ ). In contrast, three recombination events were identified in the genome of transformant $67 \mathrm{~S} 1$. The first event was the replacement of a $50.5 \mathrm{kbp}$ fragment with a $56.7 \mathrm{kbp}$ fragment that included the serotype 3 capsular genes as well as the nearby pbp $1 a$ and $p b p 2 x$ genes (Fig. $3 \mathrm{~b}$ ) that confer resistance to beta-lactam antibiotics ${ }^{23}$. The other two recombination events identified in 67S1 were distant to the capsular locus: one event of $4.9 \mathrm{kbp}$ (Fig. S2) included the liaFSR genes that sense cell envelope stress ${ }^{24}$, and the other event of $10.2 \mathrm{kbp}$ (Fig. S3) included the nanB gene associated with cleavage of host sialic acid ${ }^{25}$.

NESp transformants express functional capsule that aids in persistence during bacteremia. Flow cytometry was used to verify functional capsule production in 67S1 and 41S3 transformants. Encapsulated transformants bound to antibodies specific to the Type 3 capsule (Fig. 4a), indicating functional capsule production. We then wanted to determine if the capsule was providing NESp with a protective effect by shielding pneumococci from immune effectors present in the blood. The main mechanism of clearance during bacteremia is activation of the host innate complement system and deposition of C3b on the surface of invad- 
a

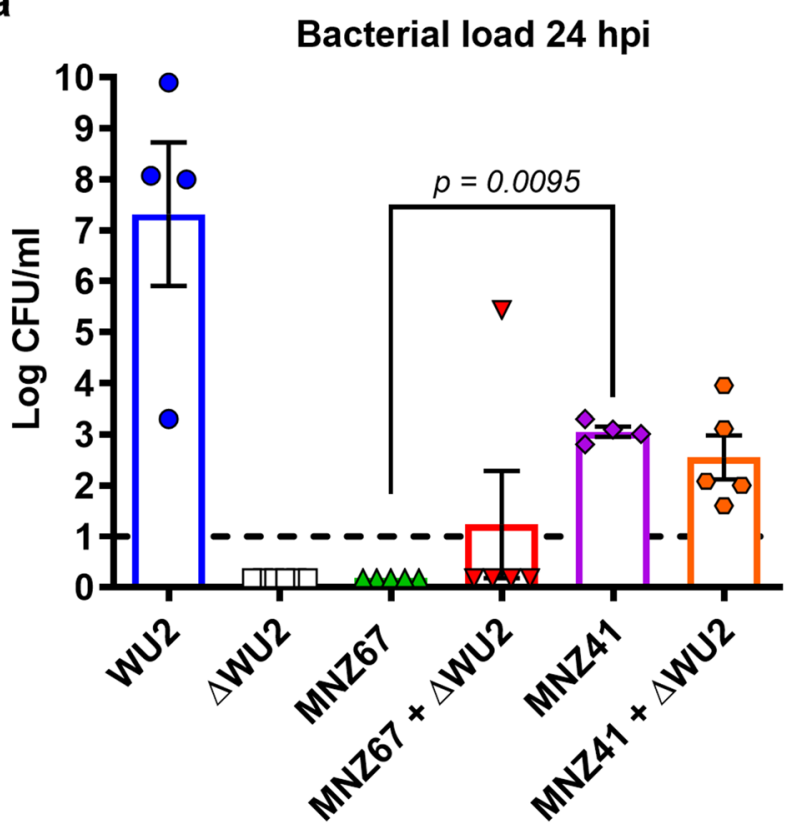

b

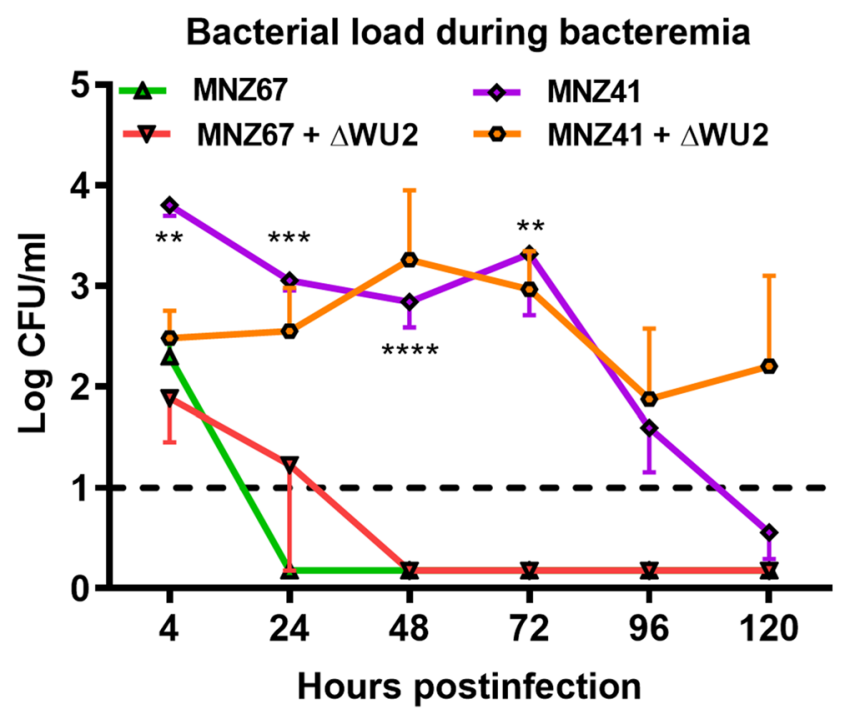

Figure 2. Pneumococcal CFUs recovered from the blood of infected mice. Seven- week-old mice were challenged with $10^{8}$ colony forming units (CFUs). Bacterial loads at $24 \mathrm{~h}$ post infection (a) and during the indicated time course (b) were estimated by plating blood mixtures on blood agar plates. At 24 hpi, significantly higher bacterial loads $(p<0.01)$ were recovered from MNZ41-infected mice compared to MNZ67-infected mice (One-Way ANOVA with Tukey's post-hoc analysis). From 4-72 hpi, MNZ41-infected mice had significantly higher $(p<0.01)$ bacterial loads compared to MNZ67-infected mice regardless of co-administration of heatkilled WU2 (Two-Way ANOVA with Tukey's post-hoc analysis). Data shown represent at least two independent experiments. Dashed line indicates the limit of detection. Error bars represent standard error of the means. ${ }^{* *} p<0.01,{ }^{* *} p<0.001,{ }^{* * *} p<0.0001$ vs MNZ67.

ing pneumococci, which results in subsequent opsonophagocytosis by immune cells ${ }^{26,27}$. The amount of C3b deposited on the surface of MNZ67 was significantly greater $(p<0.05)$ than the amount of C3b deposited on the surface of the capsule transformant $67 \mathrm{~S} 1$ (Fig. 4b). Contrarily, there was no significant difference between the amount of C3b deposited on the surface of MNZ41 when compared to its capsule transformant 41S3 (Fig. 4b).

\section{Discussion}

The main findings of this study were that NESp strains are highly transformable and able to acquire large DNA segments that enhance NESp persistence and virulence during invasive disease. When compared to rigorously optimized transformation protocols for encapsulated pneumococci that resulted in a $1 / 1000$ transformation frequency ${ }^{28}$, both carriage isolates MNZ67 and MNZ41 were highly transformable with approximately 1 positive transformant identified out of every 500 viable cells. During pneumococcal transformation, natural competence is induced through a quorum-sensing mechanism involving peptide pheromones that have been identified as two isoforms, competence-stimulating peptide (Csp)-1 and Csp-2 29,30 . MNZ67 required only Csp-1 while MNZ41 required both Csp-1 and Csp-2 for transformation. Based on sequence analysis searching for disruptions or duplications of competence genes, there was no potential explanation for the necessity of both Csp isoforms during MNZ41 transformation. However, MNZ41 encodes aliC and aliD, which are oligopeptide binding lipoproteins (OBLs) and homologues of aliA and $a l i B^{9,31}$. AliA, AliB, and AliC have been shown to impact competence induction, though the mechanism has not been fully elucidated ${ }^{30,32}$. It is possible that oligopeptide import involving these OBLs could be altering the quorum sensing or downstream signaling events that occur during competence induction. Further studies are needed to fully understand the impact of imported oligopeptides on the natural competence of pneumococci.

Moreover, capsule expression corresponded with bacterial burden and mortality during murine bacteremia. MNZ67 was rapidly cleared when there was no evidence of capsule acquisition in vivo. However, high mortality was associated with mice infected with capsule-transformed MNZ67. Nonetheless, capsule expression by transformed MNZ41 did not have as large an impact on MNZ41 virulence. MNZ41 was able to replicate in the blood through 4 dpi before being cleared; however, capsule acquisition did permit persistence of transformed MNZ41 in the blood for a longer duration. This result was consistent with our previous demonstration that MNZ41 can replicate in whole blood and resist immune effectors ${ }^{33}$. Furthermore, we have previously shown that MNZ41 expresses a choline binding protein variant that counteracts complement deposition ${ }^{33}$. Thus, it seems MNZ41 has already evolved virulence mechanisms that compensate for the lack of a protective capsule. Contrarily, encapsulation of MNZ67 transformant 67S1 significantly decreased C3b deposition, suggesting an increased pathogenic potential of the transformed MNZ67 strain. Importantly, all randomly sampled colonies isolated 
a
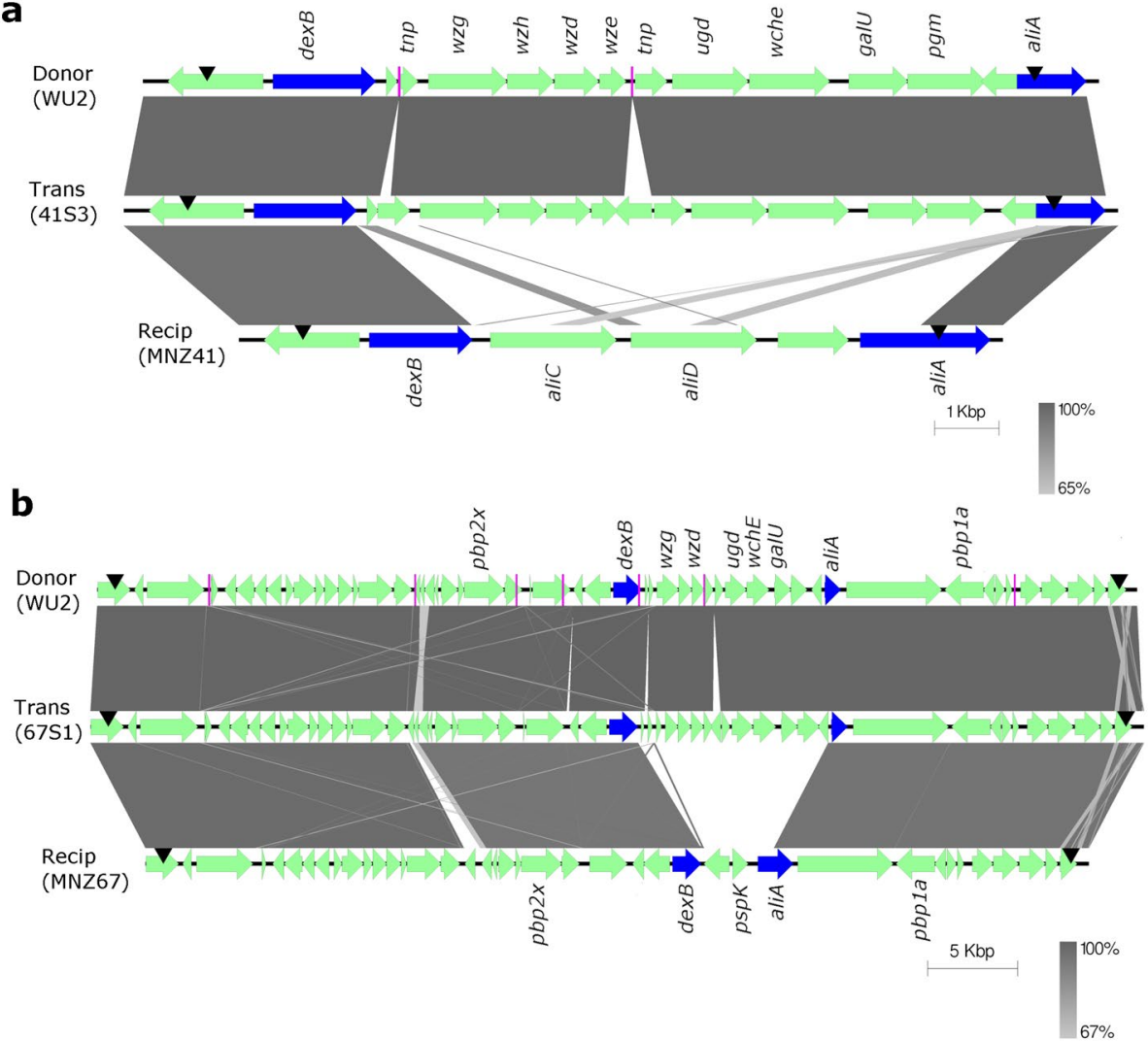

Figure 3. Genomic comparison of recombination regions involving capsule locus among donor, transformant, and recipient. For both recombination events $(\mathbf{a}, \mathbf{b})$, encapsulated serotype 3 S. pneumoniae strain WU2 served as the donor strain. (a) NESp strain MNZ41 is the recipient and 41S3 is the transformant. (b) NESp strain MNZ67 is the recipient and 67S1 is the transformant. Trans=Transformant, Recip= Recipient. Arrows indicate direction of open reading frame (ORF). Blue arrows indicate either dexB or aliA capsule-flanking genes to demarcate the capsule region. Magenta lines represent contig breakpoints. Black pointers represent recombination breakpoints. Grayscale represents \% nucleotide identity between sequences.

during bacteremia were resistant to the same antibiotics as parent NESp strains (Table 1). Therefore, NESp were able to acquire capsule and maintain antibiotic resistance during in vivo transformation.

Enhanced virulence and mortality associated with transformed MNZ67 could also be attributed to the recombination events occurring in the strain. Transfer of a $56.7 \mathrm{kbp}$ fragment into the capsular locus of MNZ67 is a recombination event length at the upper end of what has been reported for the pneumococcus ${ }^{34}$. This event included recombination of beta-lactam resistance genes encoded adjacent to the capsular locus. Additionally, recombination events identified outside of the capsule locus included genes associated with cell envelope stress and cleavage of host sialic acid by a pneumococcal neuraminidase, which could also aid in persistence and enhanced virulence of this strain. Notably, the pneumococcal neuraminidase NanA has become an increasingly popular vaccine candidate ${ }^{35}$. However, we provide evidence that recombination involving the nanA locus occurs under conditions modeling a systemic infection.

Overall, these findings support our hypothesis that NESp can acquire capsule from an encapsulated strain during bacteremia and transform into antibiotic resistant and more virulent strains. As nonencapsulated and encapsulated pneumococci continue to intimately interact during colonization, the potential for emergence of recombined strains will continue to increase. Current vaccine and treatment strategies will further select these strains that threaten treatment and survival outcomes. Surprisingly, capsule acquisition did not enhance virulence of strain MNZ41. Thus, our findings also indicate that NESp have acquired virulence mechanisms that compensate for lack of capsule and permit persistence during invasive disease.

\section{Materials and methods}

Bacterial growth and antibiotic susceptibility. S. pneumoniae strains were grown at $37{ }^{\circ} \mathrm{C}$ with $5 \%$ $\mathrm{CO}_{2}$ on blood agar (BA) containing $5 \mu \mathrm{g} / \mathrm{ml}$ gentamycin or in Todd-Hewitt broth supplemented with $0.5 \%$ yeast extract (THY). To determine antibiotic susceptibility, bacteria were grown on BA containing $50 \mu \mathrm{g} / \mathrm{ml}$ trimethoprim (Tmp), $0.3 \mu \mathrm{g} / \mathrm{ml}$ erythromycin (Erm), or $100 \mu \mathrm{g} / \mathrm{ml}$ streptomycin. A table of strains used in this study and relative phenotypic characteristics of strains are listed in Table 1. 

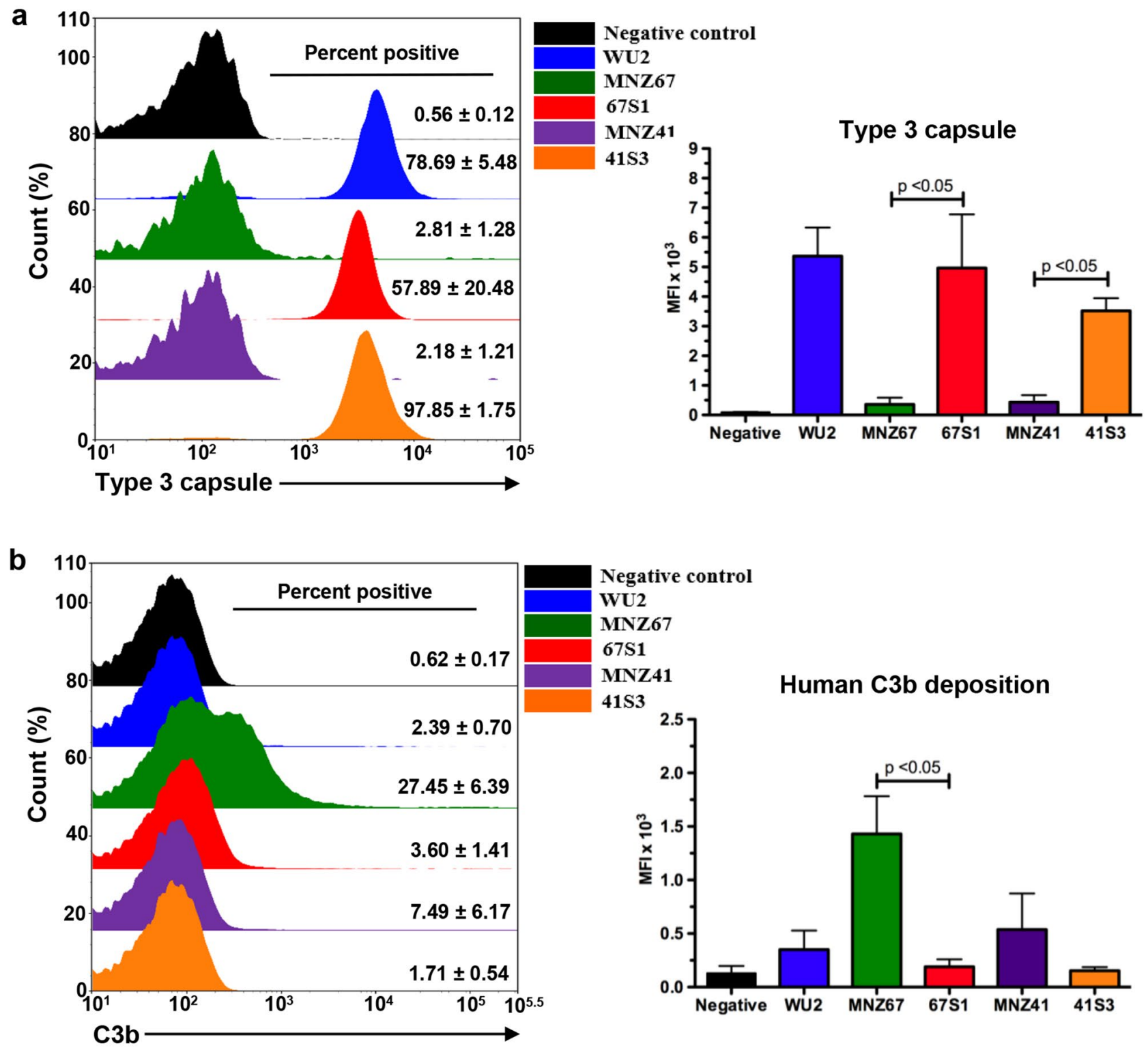

Negative control

WU2

MNZ67

67S1

MNZ41

4153

Human C3b deposition

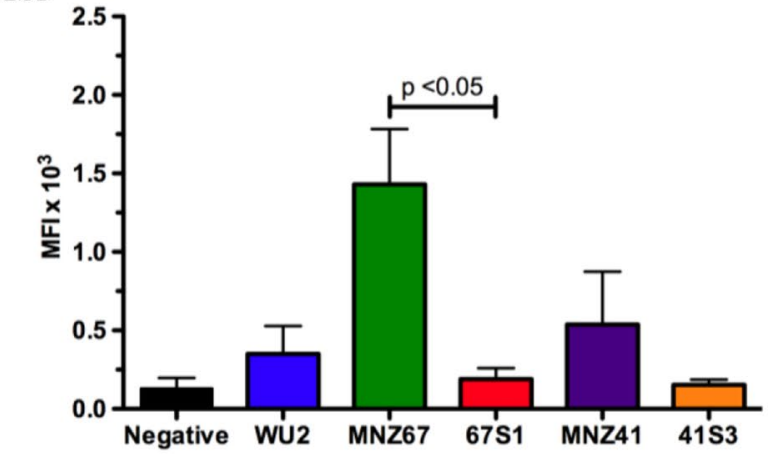

Figure 4. Flow cytometry evaluating functional capsule production and deposition of $\mathrm{C} 3 \mathrm{~b}$ on the surface of viable pneumococci. Pneumococci were incubated with antibodies against Type 3 capsule (a) or normal human serum followed by antibodies against complement component C3b (b). Cells were subsequently stained with Alexa Fluor 488 and subjected to flow cytometry. Percent positive cells were calculated by gating fluorescence higher than background (black line = gate), and averages of percent positive cells \pm standard error of the mean are listed to the right of representative histograms in overlays. Mean fluorescent intensities (MFIs) are represented in bar graphs. Data represent at least two independent experiments with 100,000 events collected for each sample. Error bars denote standard error of the mean.

In vitro transformation of NESp. Pneumococci were preconditioned in competence media (10 ml THY supplemented with $500 \mu \mathrm{l}$ of $4 \%$ bovine serum albumin, $100 \mu \mathrm{l}$ of $20 \%$ glucose, and $20 \mu \mathrm{l}$ of $10 \% \mathrm{CaCl}_{2}$ ) by culturing cells to mid-log phase $\left(\mathrm{OD}_{600} \sim 0.2\right)$ at $37^{\circ} \mathrm{C}$ with $5 \% \mathrm{CO}_{2}$. Preconditioned cultures were diluted 1:100 in fresh competence media and incubated with $2 \mu \mathrm{l}$ of $0.1 \mathrm{mg} / \mathrm{ml}$ competence-stimulating peptide- 1 (Csp-1), competence-stimulating peptide-2 (Csp-2), or both Csp-1 and Csp-2 for $12 \mathrm{~min}$ in a $37^{\circ} \mathrm{C}$ water bath. Donor DNA (500 ng genomic LEK06 prepared with Qiagen DNeasy kit) was added to $100 \mu \mathrm{l}$ of stimulated cells and incubated in a $30{ }^{\circ} \mathrm{C}$ water bath for $20 \mathrm{~min}$. After allowing contact of exogenous DNA and bacteria, mixtures were diluted 1:100 in fresh competence media and allowed to expand in a $37^{\circ} \mathrm{C}$ water bath for $1.5 \mathrm{~h}$ before plating on selective BA. Plates were incubated overnight at $37^{\circ} \mathrm{C}$ with $5 \% \mathrm{CO}_{2}$ before selecting transformants.

Murine systemic infection. Seven-week-old C57BL/6 mice were injected intraperitoneally with $10^{8}$ colony forming units (CFUs) of serotype 3 encapsulated strain WU2, heat-inactivated WU2 ( $\triangle W U 2$ ), nonencapsulated S. pneumoniae (NESp) strain MNZ67, NESp strain MNZ41, or a combination of $\Delta$ WU2 with MNZ41 or 
MNZ67. Blood samples were collected at $4 \mathrm{~h}$ postinfection, followed by sampling every $24 \mathrm{~h}$ until 8 days postinfection. Blood mixtures were serially diluted and plated on BA to enumerate pneumococcal CFU and determine recovered phenotypes. Up to ten random colonies from each plated blood sample were quadrant streaked onto selective blood agar plates to determine antibiotic susceptibility. Ten capsule-transformed colonies (five from MNZ41-infected mice and five from MNZ67-infected mice) were expanded in THY broth and saved for downstream analysis. Murine physical conditions and survival was monitored throughout experimental bacteremia by licensed veterinarians. Animal procedures were performed in accordance with protocols reviewed and approved by the Institutional Animal Care and Use Committee of the University of Mississippi Medical Center.

Polymerase chain reaction (PCR) amplification. PCR amplification was used to determine the presence or absence of genes located in the capsule polysaccharide biosynthetic (cps) locus with primer sets previously published ${ }^{36}$. Genomic DNA was isolated using a DNeasy kit (Qiagen). To verify capsule gene acquisition, primers specific for the conserved gene $c p s A$ were used, and WU2 genomic DNA served as a positive control for $c p s A$ amplification. Primers specific to NESp genes $p s p K$, aliC, and aliD were also used to determine if capsule acquisition resulted in loss of NESp genes encoded in the same locus as cpsA. MNZ67 genomic DNA was used as a positive control for $p s p K$ amplification, and MNZ41 genomic DNA was used as a positive control for aliC and aliD amplifications. Nuclease-free water was added in place of template as a negative control for amplification using all primer sets. PCR products were amplified using GoTaq DNA polymerase (Promega) with an annealing temperature of $52^{\circ} \mathrm{C}$ and cycling parameters recommended by Promega. Relative sizes of the PCR products were verified using gel electrophoresis and ethidium bromide staining.

Genome sequencing and analysis. Encapsulated donor strain WU2 and NESp recipient strain MNZ41 were previously sequenced, and available reads and assemblies were downloaded from public databases: BioProject accessions PRJEB3084 (ERR326358) for WU2 and PRJNA196249 for MNZ41. NESp recipient strain MNZ67 and capsule transformants 41S3 and 67S1 were sequenced on an Illumina MiSeq as described previously ${ }^{37}$ with the exception that $300 \mathrm{bp}$ paired-end libraries were sequenced. The new sequences were assigned BioProject accession PRJNA450976. Sequences were adapter and quality-trimmed with BBMap ${ }^{38}$, and assembled de novo as done previously ${ }^{37}$. MAUVE ${ }^{39}$ v2.3.1 was used to align and reorder the contigs of the assemblies of the recipient strains to the completely sequenced NESp reference strain $110.58^{40}$ (GenBank accession CP007593). The reads from the donor, recipients, and transformants were then mapped to the reordered contigs of the recipients with $\mathrm{BWA}^{41} \mathrm{v} 0.7 .12$, coordinate-sorted and deduplicated with Picard ${ }^{42} \mathrm{v} 1.141$, and realigned around short insertiondeletion polymorphisms (indels) with $\mathrm{GATK}^{43} \mathrm{v} 2.8-1$. Variants were called with the UnifiedGenotyper walker of GATK, and biallelic single nucleotide polymorphisms (biSNPs) were quality-filtered with BCFtools ${ }^{44}$ v1.9. $^{.}$ Recombination events were characterized with a two-step procedure that included: (1) identification of the events by following biSNPs in the core genome that are shared among the donor, recipients, and transformants, and (2) blast $\mathrm{N}$ alignment of the corresponding portions of the assemblies, which includes accessory sequences that are not shared by all strains, and visualization with Easyfig ${ }^{45}$ v2.2.2. Three or more consecutive biSNPs where the donor and transformants shared the same alternate allele compared to the recipients were considered to be part of the same recombination event. Those biSNPs identified from the self-mapping of recipient reads against the recipient assemblies were considered to be ambiguous and ignored in determining consecutive biSNPs. This analysis was performed separately with the sequences of the two in vivo transformation experiments.

Flow cytometry. Flow cytometric analysis was used to confirm functional capsule production and evaluate phenotypic variances occurring after capsule acquisition in transformants. In all experiments, $10^{7}$ pneumococci were grown to mid-log phase, collected by centrifugation, and washed with phosphate-buffered saline (PBS). To evaluate capsule production, pneumococci were incubated on ice for $30 \mathrm{~min}$ with $200 \mu \mathrm{l}$ monoclonal mouse anti-Type 3 capsule antibody Hyp3M6 (IgM isotype, diluted 1:20, kindly provided by Dr. Moon Nahm, University of Alabama at Birmingham, Birmingham, Alabama, USA). Bacterial cells were then collected by centrifugation, washed three times with PBS, suspended in $200 \mu \mathrm{l}$ of $1 \mu \mathrm{g} / \mathrm{ml}$ biotinylated goat anti-mouse IgM antibody (Southern Biotech), and incubated on ice for $30 \mathrm{~min}$. Cells incubated with PBS instead of Type 3 capsule antibody served as the negative control. To evaluate complement $\mathrm{C} 3 \mathrm{~b}$ deposition, pneumococci were incubated on ice for $30 \mathrm{~min}$ with $10 \%$ pooled normal human serum diluted in gelatin-based veronal buffer $\left(0.15 \mathrm{mM} \mathrm{CaCl}_{2}\right.$, $141 \mathrm{mM} \mathrm{NaCl}, 0.5 \mathrm{mM} \mathrm{MgCl}, 0.1 \%$ gelatin, $1.8 \mathrm{mM}$ sodium barbital, and $3.1 \mathrm{mM}$ barbituric acid, $\mathrm{pH}$ 7.3-7.4). Pneumococci were subsequently collected, washed three times with PBS, suspended in $200 \mu \mathrm{l} \mathrm{of} 1 \mu \mathrm{g} / \mathrm{ml}$ biotinylated anti-human C3b antibody (Cedarlane Laboratories Limited), and incubated on ice for $30 \mathrm{~min}$. Cells incubated with PBS instead of human serum served as the negative control. For all experiments, pneumococci were stained with streptavidin-conjugated Alexa Fluor 488 (1:1000, Invitrogen) and incubated on ice for $30 \mathrm{~min}$ in the dark. After staining, pneumococci were collected, washed four times with PBS, suspended in $500 \mu \mathrm{l}$ PBS, and analyzed by a NovoCyte flow cytometer with NovoSampler (ACEA Biosciences, Inc).

Statistics. Results were analyzed using PRISM 8 software (GraphPad Software, Inc). A student t-test was used to determine differences in mean transformation efficiencies comparing two strains. One-way analysis of variance (ANOVA) was used to evaluate if there were any overall significant differences in the means between all compared groups. Tukey posttests were performed to specifically identify the significant differences in the groups analyzed. For analysis of variance in bacterial populations over a time course, a Two-way ANOVA with Tukey posttest was performed. A p-value less than 0.05 was considered to be statistically significant. 


\section{Data availability}

Genome sequences can be accessed with accession numbers provided in the sequencing methods. The remaining data that support the findings of this study are available upon request to the corresponding author.

Received: 23 July 2020; Accepted: 16 October 2020

Published online: 03 November 2020

\section{References}

1. Kadioglu, A., Weiser, J. N., Paton, J. C. \& Andrew, P. W. The role of Streptococcus pneumoniae virulence factors in host respiratory colonization and disease. Nat. Rev. Microbiol. 6, 288-301 (2008).

2. Musher, D. M. Infections caused by Streptococcus pneumoniae: clinical spectrum, pathogenesis, immunity, and treatment. Clin. Infect. Dis. 14, 801-807 (1992).

3. Keller, L. E., Robinson, D. A. \& McDaniel, L. S. Nonencapsulated Streptococcus pneumoniae: emergence and pathogenesis. MBio 7, 1-12 (2016)

4. Pfizer. Full prescribing information. https://labeling.pfizer.com/showlabeling.aspx?id=501 (2016).

5. Merck. Prescribing information. (2015). https://www.merck.com/product/usa/pi_circulars/p/pneumovax_23/pneumovax_pi.pdf (2015).

6. Castiglia, P. Recommendations for pneumococcal immunization outside routine childhood immunization programs in Western Europe. Adv. Ther. 31, 1011-1044 (2014).

7. Centers for Disease Control and Prevention. Pneumococcal disease: Fast facts. https://www.cdc.gov/pneumococcal/about/facts .html\#ref2 (2018).

8. Geno, K. A. et al. Pneumococcal capsules and their types: past, present, and future. Clin. Microbiol. Rev. 28, 871-899 (2015).

9. Park, I. H. et al. Nontypeable pneumococci can be divided into multiple cps types, including one type expressing the novel gene pspK. MBio 3,1-11 (2012).

10. Chen, I. \& Dubnau, D. DNA uptake during bacterial transformation. Nat. Rev. Microbiol. 2, 241-249 (2004).

11. Johnston, C., Campo, N., Bergé, M. J., Polard, P. \& Claverys, J. P. Streptococcus pneumoniae, le transformiste. Trends Microbiol. 22, 113-119 (2014).

12. Li, G. et al. Addiction of hypertransformable pneumococcal isolates to natural transformation for in vivo fitness and virulence. Infect. Immun. 84, 1887-1901 (2016).

13. Chewapreecha, C. et al. Dense genomic sampling identifies highways of pneumococcal recombination. Nat. Genet. 46, 305-309 (2014).

14. Keller, L. E. et al. Immunization with pneumococcal surface protein K of nonencapsulated Streptococcus pneumoniae provides protection in a mouse model of colonization. Clin. Vaccine Immunol. 22, 1146-1153 (2015).

15. Sa-Leao, R. et al. High rates of transmission of and colonization by Streptococcus pneumoniae and Haemophilus influenzae within a day care center revealed in a longitudinal study. J. Clin. Microbiol. 46, 225-234 (2008).

16. Orihuela, C. J., Gao, G., Francis, K. P., Yu, J. \& Tuomanen, E. I. Tissue-specific contributions of pneumococcal virulence factors to pathogenesis. J. Infect. Dis. 190, 1661-1669 (2004)

17. Shenoy, A. T. \& Orihuela, C. J. Anatomical site-specific contributions of pneumococcal virulence determinants. BMC Pneumonia 8, 7 (2016).

18. Kietzman, C. C., Gao, G., Mann, B., Myers, L. \& Tuomanen, E. I. Dynamic capsule restructuring by the main pneumococcal autolysin LytA in response to the epithelium. Nat. Commun. 7, 1-9 (2016).

19. Valentino, M. D. et al. Unencapsulated Streptococcus pneumoniae from conjuntivitis encode variant traits and belong to a distinct phylogenetic cluster. Nat. Commun. 5, 5411 (2014).

20. Hyams, C., Camberlein, E., Cohen, J. M., Bax, K. \& Brown, J. S. The Streptococcus pneumoniae capsule inhibits complement activity and neutrophil phagocytosis by multiple mechanisms. Infect. Immun. 78, 704-715 (2010).

21. Park, I. H., Geno, K. A., Sherwood, L. K., Nahm, M. H. \& Beall, B. Population-based analysis of invasive nontypeable pneumococci reveals that most have defective capsule synthesis genes. PLoS ONE 9, 5-12 (2014).

22. Griffith, F. The significance of pneumococcal types. J. Hyg. (Lond) 27, 113-159 (1928).

23. Zerfaß, I., Hakenbeck, R. \& Denapaite, D. An important site in PBP2x of penicillin-resistant clinical isolates of Streptococcus pneumoniae: mutational analysis of Thr338. Antimicrob. Agents Chemother. 53, 1107-1115 (2009).

24. Eldholm, V. et al. The pneumococcal cell envelope stress-sensing system LiaFSR is activated by murein hydrolases and lipid IIinteracting antibiotics. J. Bacteriol. 192, 1761-1773 (2010).

25. Janesch, P. et al. Assessing the function of pneumococcal neuraminidases NanA, NanB and NanC in in vitro and in vivo lung infection models using monoclonal antibodies. Virulence 9, 1521-1538 (2018).

26. Winkelstein, J. A. The role of complement in the host's defense against Streptococcus pneumoniae. Rev. Infect. Dis. 3, 289-298 (1981).

27. Brown, J. S. et al. The classical pathway is the dominant complement pathway required for innate immunity to Streptococcus pneumoniae infection in mice. Proc. Natl. Acad. Sci. 99, 16969-16974 (2002).

28. Bricker, A. L. \& Camilli, A. Transformation of a type 4 encapsulated strain of Streptococcus pneumoniae. FEMS Microbiol. Lett. 172, 131-135 (1999).

29. Simon, D. et al. Competence for genetic transformation in encapsulated strains of Streptococcus pneumoniae: two allelic variants of the peptide pheromone. J. Bacteriol. 178, 6087-6090 (1996).

30. Alloing, G., Martin, B., Granadel, C. \& Claverys, J. P. Development of competence in Streptococcus pneumoniae: pheromone autoinduction and control of quorum sensing by the oligopeptide permease. Mol. Microbiol. 29, 75-83 (1998).

31. Hathaway, L. J., Meier, P. S., Bättig, P., Aebi, S. \& Mühlemann, K. A homologue of aliB is found in the capsule region of nonencapsulated Streptococcus pneumoniae. J. Bacteriol. 186, 3721-3729 (2004).

32. Hathaway, L. J. et al. Streptococcus pneumoniae detects and responds to foreign bacterial peptide fragments in its environment. Open Biol. 4, 130224 (2014).

33. Bradshaw, J. L., Pipkins, H. R., Keller, L. E., Pendarvis, J. K. \& McDaniel, L. S. Mucosal infections and invasive potential of nonencapsulated Streptococcus pneumoniae are enhanced by oligopeptide binding proteins AliC and AliD. MBio 9, e02097-e2117 (2018).

34. Cowley, L. A. et al. Evolution via recombination: Cell-to-cell contact facilitates larger recombination events in Streptococcus pneumoniae. PLoS Genet. 14, e1007410 (2018).

35. Tong, H. H., Li, D., Chen, S., Long, J. P. \& Demaria, T. F. Immunization with recombinant Streptococcus pneumoniae neuraminidase NanA protects chinchillas against nasopharyngeal colonization. Infect Immun. 73, 7775-7778 (2005).

36. Martin, C. S., Bradshaw, J. L., Pipkins, H. R. \& McDaniel, L. S. Pulmonary disease associated with nonencapsulated Streptococcus pneumoniae. Open Forum Infect Dis. 5, ofy135 (2018).

37. Keller, L. E. et al. Draft genome sequences of five multilocus sequence types of nonencapsulated Streptococcus pneumoniae. Genome Announc. 1, e00520-e613 (2013).

38. Smith, H. E. \& Yun, S. Evaluating alignment and variant-calling software for mutation identification in C. elegans by whole-genome sequencing. PLoS ONE. 12, e0174446. (2017) 
39. Rissman, A. I. et al. Reordering contigs of draft genomes using the Mauve Aligner. Bioinformatics 25, 2071-2073 (2009).

40. Hilty, M. et al. Global phylogenomic analysis of nonencapsulated Streptococcus pneumoniae reveals a deep-branching classic lineage that is dinstinct from multiple sporadic lineages. Genome Biol Evol. 6, 3281-3294 (2014).

41. Li, H. \& Durbin, R. Fast and accurate short read alignment with Burrows-Wheeler transform. Bioinformatics 25, 1754-1760 (2009).

42. Broad Institute. Picard. https://broadinstitute.github.io/picard/ (2016).

43. Mckenna, A. et al. The genome analysis toolkit : A MapReduce framework for analyzing next-generation DNA sequencing data. Genome Res. 20, 1297-1303 (2010).

44. Li, H. et al. The sequence alignment/map format and SAMtools. Bioinformatics 25, 2078-2079 (2009).

45. Sullivan, M. J., Petty, N. K. \& Beatson, S. A. Easyfig : a genome comparison visualizer. Bioinformatics 27, 1009-1010 (2011).

46. Briles, D. E. et al. Antiphosphocholine antibodies found in normal mouse serum are protective against intravenous infection with type 3 Streptococcus pneumoniae. J. Exp. Med. 153, 694-705 (1981).

\section{Acknowledgements}

We would like to acknowledge Xiao Luo for his assistance in sample preparation and sequencing of bacterial genomes. Support for this research was provided by institutional funds at the University of Mississippi Medical Center.

\section{Author contributions}

J.L.B. and L.S.M. conceived and designed the experiments. J.L.B. and L.S.M. performed the experiments. J.L.B., I.M.R., D.A.R., and L.S.M. analyzed the data. J.L.B. wrote the original manuscript. J.L.B., I.M.R., D.A.R., and L.S.M. reviewed and edited the manuscript.

\section{Competing interests}

The authors declare no competing interests.

\section{Additional information}

Supplementary information is available for this paper at https://doi.org/10.1038/s41598-020-75988-5.

Correspondence and requests for materials should be addressed to L.S.M.

Reprints and permissions information is available at www.nature.com/reprints.

Publisher's note Springer Nature remains neutral with regard to jurisdictional claims in published maps and institutional affiliations.

(c) (i) Open Access This article is licensed under a Creative Commons Attribution 4.0 International License, which permits use, sharing, adaptation, distribution and reproduction in any medium or format, as long as you give appropriate credit to the original author(s) and the source, provide a link to the Creative Commons licence, and indicate if changes were made. The images or other third party material in this article are included in the article's Creative Commons licence, unless indicated otherwise in a credit line to the material. If material is not included in the article's Creative Commons licence and your intended use is not permitted by statutory regulation or exceeds the permitted use, you will need to obtain permission directly from the copyright holder. To view a copy of this licence, visit http://creativecommons.org/licenses/by/4.0/.

(C) The Author(s) 2020 